\title{
Proposta de indicador de eficiência da gestão pública municipal em promover desenvolvimento local
}

\author{
Proposal for efficiency indicator of the municipal public management in \\ promoting local development
Propuesta de indicador de eficiencia de la gestión pública municipal en promover desarrollo local

Recebido em 06/02/2018; revisado e aprovado em 28/03/2018; aceito em 13/04/2018

DOI: http://dx.doi.org/10.20435/inter.v0i0.1831

\author{
Bruno Garcia Oliveira ${ }^{1}$ \\ Lara Bartocci Liboni
}

\begin{abstract}
Resumo: O objetivo deste artigo foi avaliar a eficiência dos munícipios do estado de São Paulo em promover desenvolvimento local. Para isso, os municípios foram agrupados em quatro clusters conforme perfil tributário e por meio da Análise Envoltória de Dados foi elaborado um indicador de eficiência. Os clusters elaborados separaram os municípios em função da dependência de repasse federal, transferência de cota de imposto estadual e arrecadação de impostos municipais. Entre os 602 municípios avaliados, somente 29 municípios foram considerados benchmarking simultâneo em todas dimensões do desenvolvimento local, sendo que 28\% destes estão na mesorregião de Campinas. Além disso, a presença de municípios benchmarking limítrofes pode indicar que a escala local ultrapassa o limite geopolítico municipal.
\end{abstract}

Palavras-chave: desenvolvimento endógeno; Data Envelopment Analysis; eficiência pública.

Abstract: The objetive of this paper were asses the municipalties eficiency at São Paulo State to improve local development. The municipalties were aggregated in four clusters by tax revenue profile. Through Data Envelopment Analysis it were created an efficiency index. The clusters elaborated separated the municipalities due to the reliance on federal transfer, transfer of state tax quota and collection of municipal taxes. Among the 602 municipalities evaluated, only 29 municipalities were considered simultaneous benchmarking in all dimensions of local development and $28 \%$ of them belongs to mesoregion of Campinas. Besides, the existence of benchmarking bordering municipalties might indicate the local scale exceeds the municipal geopolitical limit.

Keywords: endogenous development; Data Envelopment Analysis; public efficiency.

Resumen: El objetivo de este artículo fue evaluar la eficiencia de los municipios del estado de São Paulo en promover el desarrollo local. Para ello, los municipios fueron agrupados en cuatro clusters conforme perfil tributario y por medio del Análisis Envoltorio de Datos se elaboró un indicador de eficiencia. Los clusters elaborados separaron los municipios en función de la dependencia de repaso federal, transferencia de cuota de impuesto estatal y recaudación de impuestos municipales. Entre los 602 municipios evaluados, sólo 29 municipios fueron considerados benchmarking simultáneo en todas las dimensiones del desarrollo local, siendo que el $28 \%$ de estos están en la mesorregión de Campinas. Además, la presencia de municipios de benchmarking limítrofes puede indicar que la escala local sobrepasa el límite geopolítico municipal.

Palabras clave: desarrollo endógeno; Data Envelopment Analysis; eficiencia pública.

\section{INTRODUÇÃO}

O termo desenvolvimento tem ganhado importância nos últimos tempos, sendo utilizado em companhia de expressões como sustentável, local, humano e socioeconômico (MARTINS, 2002; MARTINS; VAZ; CALDAS, 2010). No Brasil, após a redemocratização na década de 1980 cresceu o enfoque no desenvolvimento a partir de uma perspectiva endógena. Reforça-se nesta

\footnotetext{
${ }^{1}$ Fundação Hermínio Ometto (FHO-Uniararas), Araras, São Paulo, Brasil.

${ }^{2}$ Universidade de São Paulo (USP), São Paulo, São Paulo, Brasil.
} 
perspectiva a importância da participação de diversos atores locais na construção de desenvolvimento conjunto (MARTINELLI; JOYAL, 2004).

No desenvolvimento local, o fator econômico passa a ser considerando em parceira com outras dimensões de desenvolvimento (WILSON, 1995; SANTOS et al., 2017). Há o empoderamento da comunidade na busca por desenvolvimento de maneira holística, focando dimensões e potencialidades econômicas, ambientais, política-institucionais e sociais de maneira integrada (NEL, 2001; BORGES, 2007).

Neste contexto, a gestão pública apresenta-se como importante ator de mudança local, sendo "emblemática para a compreensão dos entraves e das perspectivas para o desenvolvimento" (FILIPPIM; ROSSETTO; ROSSETTO, 2010, p. 736). Contudo, a avaliação de desempenho de atividades municipais ainda persiste complexa, pois "buscar padrões de gestão local e de implementação de políticas públicas e de provisão de serviços, não é tarefa fácil devido à diversidade existente entre os municípios brasileiros" (SOUZA, 2004, p. 31).

Assim, por meio da técnica de Análise Envoltória de Dados (DEA) (CHARNES; COOPER; RHODES, 1978), este artigo tem como objetivo avaliar a eficiência dos munícipios do estado de São Paulo em promover desenvolvimento local. O estado de São Paulo foi selecionado para este estudo em virtude de sua importância econômica para o Brasil, sendo responsável em 2014 por $31,2 \%$ do Valor Adicionado na economia brasileira (NOVAIS, 2017). O artigo contribui ao propor um indicador de eficiência pública utilizando múltiplas dimensões do desenvolvimento local. Além de contribuir de maneira prática com gestores municipais que podem identificar melhores práticas dos municípios considerados benchmarking e refletir sobre como a gestão pode contribuir eficientemente para o desenvolvimento de suas regiões.

\section{REFERENCIAL}

O referencial está dividido em duas partes principais. A primeira discute os conceitos de desenvolvimento exógeno e endógeno, bem como a definição de desenvolvimento local e suas dimensões. A segunda parte aborda eficiência e gestão pública, com foco em estudos anteriores sobre a temática.

\subsection{Desenvolvimento local e suas dimensões}

O desenvolvimento pode ser causado por fatores endógenos e exógenos, que determinam seu curso e direção. Este processo não ocorre de maneira isolada, usualmente sendo precedidos ou consequência de outros processos (CHOJNICKI, 2010). O modelo exógeno representa um processo "dependente" de desenvolvimento, externamente controlado. Decisões fundamentais sobre o processo de desenvolvimento (com consequentes repercussões em termos da dinâmica de empregos e modificações nas estruturas sociais) são feitos por atores econômicos externos (GAROFOLI, 2002).

O território no modelo exógeno de desenvolvimento tem papel secundário, representando apenas um conjunto de condições estáticas locais que permitem vantagens para empresas externas, como por exemplo, incentivos financeiros e baixo custo de terras (GAROFOLI, 2002).

Como alternativa ao modelo exógeno, Wilson (1995) relata uma mudança na perspectiva funcionalista de lugar. O local deixa de ser visto como receptor passivo de decisões exógenas de negócio e de atividades econômicas do capitalismo global. Neste contexto, um modelo de 
desenvolvimento endógeno é visto como aquele que garante autonomia no processo de transformação do sistema econômico local (GAROFOLI, 2002).

No modelo endógeno, "de baixo para cima", o desenvolvimento passa a ser estruturado a partir dos atores locais, e não mais somente pelo planejamento centralizado (MARTINELLI; JOYAL, 2004). Destaca-se a centralidade no processo de tomada de decisão dos atores sociais locais e sua capacidade de controlar e internalizar conhecimentos e informações externos, assumindo assim as características de um processo autossustentado de desenvolvimento (GAROFOLI, 2002).

No âmbito de um desenvolvimento endógeno, o conceito de local não alude à redução de importância, o local não deve ser visto como um espaço micro, podendo ser um município ou grupo de municípios (VAINER, 2001). O local não se refere ao tamanho, mas abarca o conjunto de relações naquele território considerando as heterogeneidades e especificidades, seja um conjunto de municípios, bairros, uma microrregião ou cidade (MARTINS; VAZ; CALDAS, 2010).

Wong (2002) aponta que a natureza holística do processo de desenvolvimento local torna complexo o entendimento das relações entre diferentes fatores socioeconômicos no processo de desenvolvimento. Para Nel (2001) desenvolvimento local é definido como processo de empoderamento da comunidade com papel definido a desempenhar e trazendo benefícios tangíveis às comunidades participantes.

Por sua vez Martins, Vaz e Caldas (2010) defendem que desenvolvimento local é composto pelas diversas dimensões de cidadania. Ele possui condições de criar um espaço que fomenta a interação entre cidadãos, recuperando a autonomia do gerir o que é público. Com base em Borges (2007), o Quadro 1 aponta as quatro dimensões de desenvolvimento local: econômico, social, ambiental e político-institucional e suas principais características.

Quadro 1 - Dimensões de desenvolvimento local

\begin{tabular}{|c|l|}
\hline Dimensão Econômica & $\begin{array}{l}\text { Incremento de operações e atividades econômicas (SUAREZ, 1993) } \\
\text { vinculada à criação, acumulação e distribuição de riqueza Gallicchio } \\
(2002)\end{array}$ \\
\hline Dimensão Social & $\begin{array}{l}\text { Relaciona-se a qualidade de vida, equidade e da integração } \\
\text { social (ZAPATA et al., 2001; GALLICCHIO, 2002). Além de relações } \\
\text { de emprego, infraestrutura social e situação geral de educação } \\
\text { (BUARQUE, 2004) }\end{array}$ \\
\hline Dimensão Ambiental & $\begin{array}{l}\text { Relação da sociedade com seus recursos naturais (SUAREZ, 1993; } \\
\text { GALLICCHIO, 2002; BUARQUE, 2004) e foco na sustentabilidade e } \\
\text { territorialização (CASAROTTO FILHO; PIRES, 2001; ZAPATA et al., } \\
\text { 2001) }\end{array}$ \\
\hline Foco na descentralização, à participação social e a garantia de \\
representação social na esfera pública (CASAROTTO FILHO; PIRES, \\
2001; ZAPATA et al., 2001; SUAREZ, 1993)
\end{tabular}

Fonte: Adaptado de Borges (2007, p. 53).

Enquanto a dimensão econômica tem foco na criação, acumulação e distribuição de riqueza, nota-se que a dimensão social tem relação com equidade, com a qualidade de vida e com aspectos da estrutura de trabalho, renda e participação. Por sua vez, a dimensão ambiental trata da relação do ser humano com o meio ambiente de forma a identificá-lo como ativo no processo de desenvolvimento ou como necessária manutenção desta relação no longo prazo. 
Além disso, há ainda a dimensão política, essencialmente relacionada à descentralização, à participação social e a garantia de representação social na esfera pública. As quatro dimensões interagem entre si e coexistem numa perspectiva sistêmica. "[...] o desenvolvimento pode ser considerado como um sistema total, com os diferentes subprocessos que possuem comportamentos diferentes entre si, mas que ao mesmo tempo influenciam e são influenciados pelo comportamento dos outros subsistemas" (BORGES, 2007, p. 54).

\subsection{Eficiência e gestão pública}

Ramos e Schabbach (2012) apontam que a avaliação da gestão pública é importante por possibilitar melhorias na eficiência do gasto público e da qualidade de gestão, além de fomentar o controle social e efetividade do Estado ao permitir a divulgação dos resultados das ações governamentais. "Para que o interesse dos munícipes seja alcançado, é dever do administrador eleito pela população gerir as finanças públicas de modo que os gastos possam fazer com que haja uma maximização do desenvolvimento" (SCARPIN; SLOMSKI, 2007, p. 921).

Cruz e Marques (2014) corroboram que a eficiência dos governos locais deve ser incentivada por promover uma "competição saudável" e buscar melhorar o desempenho econômico público. Se os gastos públicos e receitas têm o poder de afetar o desenvolvimento municipal, é função da controladoria elaborar sistemas de acompanhamento e informação sobre receitas e gastos municipais (SCARPIN; SLOMSKI, 2007).

Bogoni, Hein e Beuren (2011) reforçam que o fornecimento eficiente de serviços públicos básicos pode ser visto como meio de melhorar a qualidade de vida dos trabalhadores e, por consequência, o crescimento econômico. Para eles, "gastos em infraestrutura, educação, saúde, habitação, entre outros, que fazem parte da política fiscal local, afetam a produtividade do setor privado da economia local, pois são gastos considerados produtivos e capazes de gerar externalidades positivas" (BOGONI, HEIN; BEUREN, 2011, p. 177).

Há, contudo, algumas dificuldades inerentes ao processo de avaliação de gestão pública. Lindblad (2006) aponta que a necessidade é abordada em livros, artigos e web sites. No entanto, a avaliação de desempenho de atividades municipais ainda persiste complexa e sua utilização tem sido feita de várias maneiras.

Pesquisadores de avaliação de desempenho de governos locais tem focado em agências governamentais bem estabilizadas onde a análise é mais fácil de ser realizada (como por exemplo em departamento do corpo de bombeiros ou saneamento), enquanto pouca atenção tem sido dada à análise de desempenho para desenvolvimento econômico local (LINDBLAD, 2006).

É importante, contudo, transpor tais dificuldades à medida que avaliar eficiência e utilizar indicadores é crucial para guiar tomadores de decisão, para condução de políticas públicas e mensuração do desempenho da gestão (FRAINER et al., 2017).

Neste âmbito, a utilização da Análise Envoltória de Dados (DEA) (CHARNES; COOPER; RHODES, 1978) tem crescido como alternativa metodológica à complexidade do estudo de eficiência pública. O Quadro 2 apresenta estudos com foco na avaliação de eficiência de gestão pública. Relaciona o objetivo, o método e indicadores utilizados. 
Quadro 2 - Resumo de artigos que utilizam DEA para mensurar eficiência local

\begin{tabular}{|c|c|c|c|}
\hline AUTOR & OBJETIVO & MÉTODO & INPUT \\
\hline $\begin{array}{l}\text { Adler, } \\
\text { Yazhemsky e } \\
\text { Tarverdyan } \\
\text { (2010) }\end{array}$ & $\begin{array}{l}\text { Estimar eficiência relativa de } \\
\text { países em desenvolvimento na } \\
\text { utilização de fontes de recursos } \\
\text { para alcance dos Objetivos de } \\
\text { Desenvolvimento do Milênio }\end{array}$ & $\begin{array}{c}\text { (DEA-PCA) } \\
\text { BCC-OUTPUT }\end{array}$ & $\begin{array}{l}\text { Produto Interno Bruto, } \\
\text { Investimento Estrangeiro Direto } \\
\text { Remessa de Entrada }\end{array}$ \\
\hline Lu e Lo (2012) & $\begin{array}{c}\text { Estudar quais regiões da China } \\
\text { apresentavam mais progresso } \\
\text { em relação à outras no mesmo } \\
\text { contexto } \\
\end{array}$ & $\begin{array}{l}\text { DEA Estratificado } \\
\text { CCR-Output }\end{array}$ & Reserva de capital \\
\hline $\begin{array}{l}\text { Reig-Martínez } \\
\text { (2013) }\end{array}$ & $\begin{array}{l}\text { Calcular um Índice Composto } \\
\text { de Qualidade de Vida para } 42 \\
\text { países, pertencentes ao Espaço } \\
\text { Econômico Europeu, África do } \\
\text { Norte e Oriente Médio }\end{array}$ & $\begin{array}{c}\text { DEA- BCC em } 3 \\
\text { fases (uma delas } \\
\text { PCA) }\end{array}$ & Input virtual \\
\hline $\begin{array}{c}\text { Afonso, } \\
\text { Schuknecht e } \\
\text { Tanzi (2010) }\end{array}$ & $\begin{array}{l}\text { Analisar o o impacto dos gastos } \\
\text { públicos na distribuição de } \\
\text { renda em países da OECD, } \\
\text { considerando em particular } \\
\text { a qualidade da educação e } \\
\text { instituições públicas }\end{array}$ & DEA (BCC E CCR) & $\begin{array}{l}\text { Total do gasto público social } \\
\text { como \%PIB, em média, para o } \\
\text { período 1995- } 2000\end{array}$ \\
\hline $\begin{array}{c}\text { Murias, } \\
\text { Martinez e } \\
\text { Miguel (2006) }\end{array}$ & $\begin{array}{c}\text { Estimar um indicador sintético } \\
\text { de qualidade de vida usando } \\
\text { DEA }\end{array}$ & DEA (OUTPUT) & $\begin{array}{l}\text { Rendimento Disponível Bruto } \\
\text { (per capita) } \\
\text { Tamanho médio de habitação } \\
\text { Estoque de capital líquido total } \\
\text { Estoque de capital humano } \\
\text { Percentagem de contratos } \\
\text { de longo prazo ou por tempo } \\
\text { indeterminado }\end{array}$ \\
\hline $\begin{array}{c}\text { Faria, } \\
\text { Jannuzzi e } \\
\text { Silva (2008) }\end{array}$ & $\begin{array}{l}\text { Analisar a eficiência dos } \\
\text { gastos públicos nos municípios } \\
\text { fluminenses no } \\
\text { período } 1999 / 2000\end{array}$ & DEA- BCC (INPUT) & $\begin{array}{l}\text { Despesas per capita com } \\
\text { educação } \\
\text { Despesas per capita com cultura } \\
\text { Despesas per capita com saúde } \\
\text { e saneamento } \\
\end{array}$ \\
\hline $\begin{array}{l}\text { Afonso e } \\
\text { Fernandes } \\
\text { (2005) }\end{array}$ & $\begin{array}{c}\text { Avaliar a eficiência relativa de } \\
\text { governos locais utilizando DEA e } \\
\text { análise paramétricas }\end{array}$ & $\begin{array}{c}\text { DEA- BCC (INPUT } \\
\text { e OUTPUT) + } \\
\text { Tobit }\end{array}$ & Gasto Municipal per capita \\
\hline $\begin{array}{c}\text { Carvalho et al. } \\
\text { (2017) }\end{array}$ & $\begin{array}{c}\text { Analisar a eficiência } \\
\text { agropecuária de treze } \\
\text { municípios pertencentes ao EDR } \\
\text { de Andradina }\end{array}$ & $\begin{array}{l}\text { Agrupamentos + } \\
\text { Dea (BCC-output) }\end{array}$ & $\begin{array}{l}\text { (1)Terras com lavouras } \\
\text { permanentes; (2) Pessoal } \\
\text { ocupado em estabelecimentos } \\
\text { agropecuários; (3) Unidades } \\
\text { de máquinas e implementos } \\
\text { agrícolas }\end{array}$ \\
\hline
\end{tabular}

Fonte: Oliveira (2015, p. 89).

A definição dos inputs nos textos pesquisados costuma ocorrer em virtude do objetivo que se busca alcançar e da disponibilidade de coleta dos indicadores. Observa-se a preferência por indicadores financeiros locais ou nacionais, conforme escala em estudo.

Com relação ao método empregado, há preferência para a utilização de retorno variável de escala (BCC) e orientação para OUTPUT. Observa-se também que em alguns estudos há utilização de outros métodos conjuntamente com a Análise Envoltória de Dados. Para considerar variáveis 
não discricionárias, Afonso e Fernandes (2005) utilizam análise Tobit. Além disso, para agrupar diversos outputs Carvalho et al. (2017), Reig-Martínez (2013) e Adler, Yazhemsky e Tarverdyan (2010) utilizam análise de componentes principais (DEA-PCA).

\section{MÉTODO}

Para elaboração do indicador de Eficiência foi utilizado a técnica de a Análise Envoltória de Dados (DEA), que consiste num método de programação linear para avaliação de eficiência de entidades sem fins lucrativos participantes de programas públicos (CHARNES; COOPER; RHODES, 1978).

Para Colucci (2014, p. 85) "o grande mérito da técnica DEA é o fato de ela dispensar o estabelecimento de critérios arbitrários pelo pesquisador, já que os pesos serão atribuídos pela própria técnica por meio do conjunto de dados disponíveis". Além disso, o método é flexível ao permitir a utilização de múltiplas variáveis de inputs e outputs e gerar um único indicador de eficiência (SOUZA et.al., 2017).

Segundo Golany e Roll (1989) a utilização da DEA requer especial atenção a três aspectos: (1) a seleção das Decision Making Units (DMU); (2) a seleção de inputs e outputs do modelo e (3) a seleção da escala e orientação do modelo.

\subsection{Seleção das Decision Making Units (DMU)}

A Análise Envoltória de Dados avalia a eficiência das Unidades Tomadoras de Decisão, normalmente chamadas de Decision Make Unit (DMU). O uso deste termo enfatiza o fato que os dados não são ponderados por referências a preços de mercado e/ou taxa de desconto (CHARNES; COOPER; RHODES, 1978).

Neste estudo, os municípios do Estado de São Paulo foram selecionados como DMU devido sua importância econômica para o país, que representam juntos mais de 30\% do valor adicionado a economia brasileira no ano de 2014 (NOVAIS, 2017). A primeira etapa para definição das DMUs foi: (1) coletar nomes e população total residente em todos os municípios do estado de São Paulo, por meio da SEADE (2013); e (2) coletar dados sobre arrecadação municipal, a partir de dados do Tesouro Nacional (2013).

Como todos os municípios são do mesmo estado, mas ainda assim possuem perfis diversos, antes de elaborar o índice de eficiência deve-se atentar à questão de homogeneidade entre as DMUs (DYSON et al., 2001). Assim, com base na arrecadação, os municípios do estado foram reagrupados utilizando Análise de Cluster K-means. Segundo Tristão (2003), a partir das principais fontes de arrecadação é possível descrever o perfil do município. Os indicadores utilizados para a Análise de Cluster e o perfil dos municípios segundo Tristão (2003) constam no Quadro 3.

Quadro 3 - Perfil dos municípios conforme principal tributo de arrecadação no cluster

\begin{tabular}{|c|l|l|}
\hline $\begin{array}{c}\text { Principal fonte de } \\
\text { arrecadação do Cluster }\end{array}$ & \multicolumn{1}{|c|}{ Descrição } & \multicolumn{1}{c|}{ Perfil do Município } \\
\hline Imposto Predial e & $\begin{array}{l}\text { Imposto municipal, com fato } \\
\text { gerador a propriedade, o domínio } \\
\text { útil ou a posse de bens imóveis }\end{array}$ & $\begin{array}{l}\text { Baixa dependência de repasses, } \\
\text { situam-se em áreas turísticas das } \\
\text { regiões sul e sudeste. }\end{array}$ \\
\hline
\end{tabular}




\begin{tabular}{|c|l|l|}
\hline $\begin{array}{c}\text { Principal fonte de } \\
\text { arrecadação do Cluster }\end{array}$ & \multicolumn{1}{|c|}{ Descrição } & \multicolumn{1}{|c|}{ Perfil do Município } \\
\hline $\begin{array}{c}\text { Impostos sobre Serviços } \\
\text { de Qualquer Natureza } \\
\text { (ISSQN) }\end{array}$ & $\begin{array}{l}\text { Imposto municipal, com o fato } \\
\text { gerador a prestação por empresa } \\
\text { ou profissional autônomo de } \\
\text { serviço. }\end{array}$ & $\begin{array}{l}\text { Municípios com grande } \\
\text { concentração urbana, } \\
\text { normalmente centros } \\
\text { metropolitanos. }\end{array}$ \\
\hline $\begin{array}{c}\text { Imposto Estadual, com fato } \\
\text { gerador operações relativas à } \\
\text { Imposto sobre a } \\
\text { Circulaçãão de mercadorias e } \\
\text { e Serviços (ICMS) } \\
\text { sobre prestaçães de serviços } \\
\text { de transporte interestadual e } \\
\text { intermunicipal. Da arrecadação } \\
\text { do ICMS, 25\% é distribuída pelo } \\
\text { Estado aos seus municípios. }\end{array}$ & $\begin{array}{l}\text { São municípios com existência } \\
\text { de razoável potencial econômico, } \\
\text { dado que a cota-parte de ICMS } \\
\text { é distribuída proporcionalmente } \\
\text { ao valor adicionado criado no } \\
\text { município. }\end{array}$ \\
\hline Fundo de Participação dos \\
Municípios (FPM) & $\begin{array}{l}\text { Fundo elaborado a partir de } \\
\text { impostos federais e destinado } \\
\text { a municípios com base na } \\
\text { população residente e renda. }\end{array}$ & $\begin{array}{l}\text { São municípios pequenos, } \\
\text { predominantemente rurais e que } \\
\text { apresentam os piores resultados } \\
\text { na arrecadação de seus tributos. }\end{array}$ \\
\hline
\end{tabular}

Fonte: Tristão (2003).

\subsection{Seleção dos inputs e outputs}

A segunda etapa consistiu na definição dos indicadores utilizados na Análise Envoltória de dados. Observa-se no Quadro 2 que a mensuração de eficiência da gestão pública é usualmente feita a partir da utilização de inputs financeiros na escala em análise (gastos público, investimento, PIB e etc.). Assim, o Quadro 4 descreve os três inputs utilizados neste trabalho e a fonte de coleta de dados.

Quadro 4 - Inputs e fonte de coleta de dados

\begin{tabular}{|c|c|l|c|}
\hline NOME & \multicolumn{1}{|c|}{ CÁLCULO } & \multicolumn{1}{c|}{ DESCRIÇÃO } & FONTE \\
\hline CUSTEIO & $\begin{array}{c}\text { DESPESA DE CUSTEIO } \\
\text { NÚMERO DE HABITANTES }\end{array}$ & $\begin{array}{l}\text { Representa os gastos governamentais } \\
\text { per capita para a manutenção e } \\
\text { operacionalização da administração }\end{array}$ & $\begin{array}{c}\text { Tesouro } \\
\text { Nacional }\end{array}$ \\
\hline ORÇAMENTO & $\frac{\text { RECEITA ORÇAMENTÁRIA }}{\text { NÚMERO DE HABITANTES }}$ & $\begin{array}{l}\text { Representa o total de receitas per } \\
\text { capita disponível para execução de } \\
\text { atividades e operacionalização da } \\
\text { administração pública }\end{array}$ & $\begin{array}{c}\text { Tesouro } \\
\text { Nacional }\end{array}$ \\
\hline SERVIDOR & $\frac{\text { NÚMERO DE SERVIDOR }}{\text { NÚMERO DE HABITANTES }}$ & $\begin{array}{l}\text { Representa a quantidade de } \\
\text { pessoas per capita disponível para } \\
\text { operacionalização das atividades } \\
\text { públicas }\end{array}$ & $\begin{array}{l}\text { Instituto } \\
\text { Brasileiro de } \\
\text { Geografia e } \\
\text { Estatística }\end{array}$ \\
\hline
\end{tabular}

Fonte: Oliveira (2015).

Com relação aos outputs, foram definidos conforme as quatro dimensões de desenvolvimento local apresentadas no Quadro 1. Os indicadores utilizados em cada dimensão foram discutidos e selecionados no trabalho de Oliveira (2015). O Quadro 5 relaciona a dimensão, o indicador e a fonte de coleta de dados. 
Quadro 5 - Outputs do Modelo DEA

\begin{tabular}{|c|c|c|}
\hline DIMENSÃO & INDICADOR & FONTE DE COLETA \\
\hline SOCIAL & $\begin{array}{c}\text { Índice Firjan de Desenvolvimento } \\
\text { Municipal }\end{array}$ & Federação das Indústrias do Rio de Janeiro \\
\hline \multirow{2}{*}{ ECONÔMICO } & Índice De Gini & $\begin{array}{c}\text { Programa das Nações Unidas para o } \\
\text { Desenvolvimento }\end{array}$ \\
\hline & PIB MUNICIPAL Per Capita & $\begin{array}{c}\text { Secretaria do Planejamento e } \\
\text { Desenvolvimento Regional }\end{array}$ \\
\hline \multirow{3}{*}{$\begin{array}{l}\text { POLÍTICO- } \\
\text { INSTITUCIONAL }\end{array}$} & $\begin{array}{l}\text { Participação em Organizações Não } \\
\text { Governamentais }\end{array}$ & $\begin{array}{c}\text { Instituto Brasileiro de Geografia e } \\
\text { Estatística }\end{array}$ \\
\hline & Número de Conselhos Municipais & $\begin{array}{c}\text { Instituto Brasileiro de Geografia e } \\
\text { Estatística }\end{array}$ \\
\hline & Índice Firjan de Gestão Fiscal & Federação Das Indústrias do Rio de Janeiro \\
\hline \multirow[b]{2}{*}{ AMBIENTAL } & Índice de Avaliação Ambiental & Programa Município Verde e Azul \\
\hline & Índice de Desenvolvimento Rural & $\begin{array}{l}\text { Confederação Nacional da Agricultura e } \\
\text { Pecuária }\end{array}$ \\
\hline
\end{tabular}

Fonte: Oliveira (2015).

Cada uma das quatro dimensões de desenvolvimento local (econômica, social, ambiental e político institucional) foi avaliada separadamente em cada um dos quatro clusters, resultando assim em 16 modelos de eficiência. Os municípios que alcançaram eficiência simultânea nas quatro dimensões avaliadas foram então considerado benchmarking geral.

\subsection{Seleção da escala e orientação do modelo}

O modelo adotado neste trabalho foi o com retorno variável de escala e orientação para output (BCC-OUTPUT). A orientação para Output foi recorrente nos estudos que constam no Quadro 2. A fórmula a seguir apresenta o modelo de programação linear para BCC orientado aos produtos.

$$
\begin{aligned}
& \text { Min } h_{0}=\sum_{i=1}^{n} v_{i} x_{i o}+k \\
& \text { Sujeito a } \sum_{r=1}^{m} u_{r} y_{r o}=1 \\
& \quad \sum_{i=1}^{n} v_{i} x_{i j}+k-\sum_{r=1}^{m} u_{r} y_{r j} \geq 0 \\
& \text { para } u_{r} \geq 0, v_{i} \geq 0, k \in \mathfrak{k e} \\
& \quad i=1, \ldots, n ; r=1, \ldots, m \text { e } j=1, \ldots, N .
\end{aligned}
$$

Neste contexto, as DMUs que estarão no envoltório, consideradas eficientes em relação às demais, serão aquelas que, a partir do nível atual de gasto, obtiverem os melhores resultados, sem ter que (1) aumentar um ou mais input e/ou (2) reduzir algum de seus outputs.

Por fim, são realizadas análises descritivas dos índices de eficiência gerados em especial para a caracterização das mesorregiões do estado. Os dados foram então analisados por meio de gráficos e tabelas. Para análises foi utilizado IBM SPSS STATISTICS 20 e R Project. 


\section{RESULTADOS E DISCUSSÕES}

Os resultados e discussões estão divididos em dois blocos. O primeiro discute o resultado obtido com o agrupamento dos municípios e sua distribuição nas mesorregiões do estado. A segunda parte se dedica especificamente ao indicador de eficiência e municípios benchmarking.

\subsection{Agrupamentos}

Segundo Golany e Roll (1989), pela característica metodológica do DEA é necessário atenção à homogeneidade das DMUs em análise. Para este estudo, foram utilizados os mesmos indicadores proposto por Tristão (2003), a Tabela 1 apresenta o centro dos Clusters obtidos. Ressalta-se que do total de 645 municípios do estado, a base de dados final foi composta 602 municípios, em virtude de alguns municípios não possuírem informação completa (missing values).

Em comparação ao trabalho de Tristão (2003), também foi possível identificar dois grupamentos dependentes das transferências governamentais. Especificamente o Cluster III para o FPM $(0,4948)$ e o Cluster IV para a quota parte do ICMS $(0,43)$. Contudo, pelo perfil econômico do estado, o nível de repasse da quota parte de ICMS se manteve estável nos demais grupamentos (em torno de 0,23).

Tabela 1 - Centros dos Agrupamentos para Municípios Paulistas no ano de 2010

\begin{tabular}{lcccc} 
& Dependência Mista & Arrecadadores & Dependência FPM & Dependência ICMS \\
\hline IPTU & 0,022 & 0,071 & 0,007 & 0,024 \\
ISSQN & 0,038 & 0,071 & 0,018 & 0,049 \\
ICMS & 0,260 & 0,221 & 0,244 & 0,436 \\
FPM & 0,301 & 0,143 & 0,495 & 0,194 \\
\hline
\end{tabular}

Fonte: Elaborado pelo Autor

No entanto, para os demais grupamentos os resultados foram diferentes de Tristão (2003). O segundo cluster agrupou os municípios com melhor desempenho de arrecadação tanto para IPTU, quanto para ISSQN. Há certa dependência do repasse da quota parte de ICMS, mas este grupo apresenta os melhores arrecadadores de tributos municipais.

Por fim, o quarto agrupamento apresenta um nível de dependência mista. Estes municípios não possuem bons resultados de arrecadação de tributos municipais e tem dependência do repasse feito pelo FPM $(0,2597)$. Contudo, por apresentarem certa atividade industrial também possuem parte de seu perfil tributário composto pela quota parte do ICMS $(0,3007)$. Assim, os municípios foram divididos em quatro grupos, conforme Tabela 4.

Entre os quatro grupos, o maior em número de integrantes foi o Cluster I - Dependência Mista com 43\% do total analisado. Já o Cluster III - Dependência do FPM, são municípios pequenos com população mediana de 3462 habitantes e o município mais populoso com 6163 habitantes. Com intuito de verificar a distribuição destes municípios no Estado, foi elaborada a Tabela 2.

A tabela apresenta a distribuição de municípios em função das Mesorregiões do Estado. Nota-se que $76 \%$ dos municípios da região metropolitana de São Paulo foram classificados como Arrecadadores, tal fato corrobora a classificação anteriormente proposta por Tristão (2003) ao apontar que municípios com arrecadação de ISSQN normalmente estão em regiões metropolitanas. 
Tabela 2 - Distribuição dos clusters por Mesorregião

\begin{tabular}{lcccc} 
& $\begin{array}{c}\text { Dependência } \\
\text { Mista }\end{array}$ & \begin{tabular}{c} 
Arrecadadores \\
\cline { 2 - 4 }
\end{tabular} & $\begin{array}{c}\text { Dependência } \\
\text { FPM }\end{array}$ & $\begin{array}{c}\text { Dependência } \\
\text { ICMS }\end{array}$ \\
\hline Araçatuba & $46 \%$ & $11 \%$ & $23 \%$ & $20 \%$ \\
Araraquara & $58 \%$ & $11 \%$ & $5 \%$ & $26 \%$ \\
Assis & $45 \%$ & $13 \%$ & $29 \%$ & $13 \%$ \\
Bauru & $49 \%$ & $20 \%$ & $18 \%$ & $13 \%$ \\
Campinas & $30 \%$ & $47 \%$ & $9 \%$ & $15 \%$ \\
Itapetininga & $49 \%$ & $16 \%$ & $27 \%$ & $8 \%$ \\
Litoral Sul Paulista & $67 \%$ & $20 \%$ & $0 \%$ & $13 \%$ \\
Macro Metropolitana Paulista & $31 \%$ & $53 \%$ & $3 \%$ & $13 \%$ \\
Marília & $35 \%$ & $15 \%$ & $40 \%$ & $10 \%$ \\
Metropolitana de São Paulo & $12 \%$ & $76 \%$ & $0 \%$ & $12 \%$ \\
Piracicaba & $42 \%$ & $46 \%$ & $4 \%$ & $8 \%$ \\
Presidente Prudente & $50 \%$ & $8 \%$ & $33 \%$ & $10 \%$ \\
Ribeirão Preto & $45 \%$ & $29 \%$ & $6 \%$ & $19 \%$ \\
São José do Rio Preto & $45 \%$ & $10 \%$ & $38 \%$ & $7 \%$ \\
Vale do Paraíba Paulista & $41 \%$ & $38 \%$ & $13 \%$ & $8 \%$ \\
\hline
\end{tabular}

Fonte: Elaborado pelo autor

\subsection{Análise Envoltória de Dados}

Para cada cluster foram executados quatro análises em função das dimensões de desenvolvimento local, resultando assim em 16 rankings de eficiência realizado. A Tabela 3 apresenta os resultados obtidos com a Análise Envoltória de Dados para cada um dos modelos em cada cluster de análise.

Tabela 3 - Indicadores de Eficiência DEA - Modelo Geral

Indicadores de Eficiência DEA - Modelo Geral

\begin{tabular}{clrrrrrrrr}
\hline & & \multicolumn{2}{c}{ SOCIAL } & \multicolumn{2}{c}{ ECONÔMICO } & \multicolumn{2}{c}{ POLÍTICO } & \multicolumn{2}{c}{ AMBIENTAL } \\
\hline & Eficientes & 15 & $6 \%$ & 21 & $8 \%$ & 37 & $14 \%$ & 35 & $14 \%$ \\
& $1<\mathrm{F}=<1.1$ & 82 & $32 \%$ & 22 & $8 \%$ & 49 & $19 \%$ & 56 & $22 \%$ \\
Cluster I- & $1.1<\mathrm{F}=<1.2$ & 115 & $44 \%$ & 49 & $19 \%$ & 45 & $17 \%$ & 63 & $24 \%$ \\
Dependência Mista & $1.2<\mathrm{F}=<1.3$ & 43 & $17 \%$ & 63 & $24 \%$ & 38 & $15 \%$ & 42 & $16 \%$ \\
& $1.3<\mathrm{F}=<1.5$ & 4 & $2 \%$ & 74 & $29 \%$ & 49 & $19 \%$ & 45 & $17 \%$ \\
& $1.5<\mathrm{F}$ & 0 & 0 & 30 & $12 \%$ & 41 & $16 \%$ & 18 & $7 \%$ \\
\hline \multirow{3}{*}{ Cluster II- } & Eficiente & 15 & $10 \%$ & 14 & $9 \%$ & 29 & $19 \%$ & 26 & $17 \%$ \\
Arrecadadores & $1<\mathrm{F}<=1.1$ & 71 & $47 \%$ & 22 & $15 \%$ & 22 & $15 \%$ & 40 & $27 \%$ \\
& $1.1<\mathrm{F}<=1.2$ & 41 & $27 \%$ & 45 & $30 \%$ & 29 & $19 \%$ & 41 & $27 \%$ \\
& $1.2<\mathrm{F}<=1.3$ & 19 & $13 \%$ & 42 & $28 \%$ & 23 & $15 \%$ & 19 & $13 \%$ \\
& $1.3<\mathrm{F}<=1.4$ & 3 & $2 \%$ & 20 & $13 \%$ & 17 & $11 \%$ & 17 & $11 \%$ \\
& $1.4<\mathrm{F}<=1.5$ & 1 & $1 \%$ & 4 & $3 \%$ & 9 & $6 \%$ & 5 & $3 \%$ \\
& $\mathrm{~F}>1.5$ & 0 & $0 \%$ & 3 & $2 \%$ & 21 & $14 \%$ & 2 & $1 \%$ \\
\hline
\end{tabular}


Indicadores de Eficiência DEA - Modelo Geral

\begin{tabular}{clllllllll}
\hline & & \multicolumn{2}{c}{ SOCIAL } & \multicolumn{2}{c}{ ECONÔMICO } & \multicolumn{2}{c}{ POLÍTICO } & \multicolumn{2}{c}{ AMBIENTAL } \\
\hline & Eficiente & 16 & $13 \%$ & 23 & $19 \%$ & 26 & $22 \%$ & 23 & $19 \%$ \\
& $1<\mathrm{F}<=1.1$ & 41 & $34 \%$ & 20 & $17 \%$ & 18 & $15 \%$ & 25 & $21 \%$ \\
Cluster III- & $1.1<\mathrm{F}<=1.2$ & 51 & $43 \%$ & 30 & $25 \%$ & 35 & $29 \%$ & 32 & $27 \%$ \\
Dependência FPM & $1.2<\mathrm{F}<=1.3$ & 11 & $9 \%$ & 20 & $17 \%$ & 12 & $10 \%$ & 15 & $13 \%$ \\
& $1.3<\mathrm{F}<=1.4$ & & & 12 & $10 \%$ & 12 & $10 \%$ & 12 & $10 \%$ \\
& $1.4<\mathrm{F}<=1.5$ & & & 2 & $2 \%$ & 3 & $3 \%$ & 8 & $7 \%$ \\
& $\mathrm{~F}>1.5$ & & & 12 & $10 \%$ & 13 & $11 \%$ & 4 & $3 \%$ \\
\hline Cliciente & 10 & $14 \%$ & 13 & $18 \%$ & 18 & $24 \%$ & 20 & $27 \%$ \\
Dependência ICMS & $1<\mathrm{F}<=1.1$ & 29 & $39 \%$ & 15 & $20 \%$ & 11 & $15 \%$ & 18 & $24 \%$ \\
& $1.1<\mathrm{F}<=1.2$ & 23 & $31 \%$ & 23 & $31 \%$ & 10 & $14 \%$ & 14 & $19 \%$ \\
& $1.2<\mathrm{F}<=1.3$ & 8 & $11 \%$ & 14 & $19 \%$ & 15 & $20 \%$ & 14 & $19 \%$ \\
& $1.3<\mathrm{F}<=1.4$ & 4 & $5 \%$ & 3 & $4 \%$ & 7 & $9 \%$ & 2 & $3 \%$ \\
& $1.4<\mathrm{F}<=1.5$ & & & 3 & $4 \%$ & 6 & $8 \%$ & 3 & $4 \%$ \\
& $\mathrm{~F}>1.5$ & & & 3 & $4 \%$ & 7 & $9 \%$ & 3 & $4 \%$ \\
\hline
\end{tabular}

Fonte: Elaborado pelo autor

Em linhas gerais, o modelo voltado para mensurar eficiência da gestão pública na dimensão social foi o que obteve as menores proporções de DMUs eficientes com resultados variando entre $6 \%$ e $14 \%$ nos clusters. Na dimensão Econômica, o modelo com melhor resultado de eficiência foi o obtido no Cluster III, seguido pelo Cluster IV. Na dimensão política e ambiental o Cluster IV obteve as maiores proporções de municípios eficientes, respectivamente com $24 \%$ e $27 \%$.

Do total de DMUs, 29 municípios foram definidos como eficientes em todas as quatro dimensões analisadas. Estes municípios foram considerados como benchmarking geral, pois em cada cluster a que pertenciam alcançaram eficiência simultânea nas quatro dimensões de desenvolvimento. O número absoluto de benchmarking geral por cluster se manteve estável entre 6 e 8 municípios conforme Tabela 4

Tabela 4 - Porcentagem de Benchmarking Geral por Cluster

\begin{tabular}{lccc}
\multicolumn{1}{c}{ Tipologia } & $\begin{array}{c}\text { Quantidade por } \\
\text { Cluster }\end{array}$ & $\begin{array}{c}\text { Benchmarking } \\
\text { Geral }\end{array}$ & $\begin{array}{c}\text { Porcentagem de } \\
\text { Benchmarking por Cluster }\end{array}$ \\
\hline Cluster I - Dependência Mista & 259 & 7 & $3 \%$ \\
Cluster II - Arrecadadores & 150 & 8 & $5 \%$ \\
Cluster III - Dependência do FPM & 119 & 8 & $7 \%$ \\
Cluster IV- Dependência da cota & 74 & 6 & $8 \%$ \\
ICMS & 602 & 29 & $5 \%$
\end{tabular}

Fonte: Elaborado pelo autor

Geograficamente os municípios benchmarking estão distribuídos nas dez mesorregiões do Estado de São Paulo. Contudo, há predominância de municípios benchmarking geral na Mesorregião de Campinas (conforme Tabela 5). Assim, a mesorregião de Campinas apresenta-se como destaque da gestão pública na promoção de desenvolvimento local. 
Tabela 5 - Benchmarking geral por Mesorregião

\begin{tabular}{|c|c|c|c|c|c|}
\hline Mesorregião & Quantidade & $\%$ & Mesorregião & Quantidade & $\%$ \\
\hline Campinas & 8 & $28 \%$ & Ribeirão Preto & 2 & $7 \%$ \\
\hline $\begin{array}{l}\text { Metropolitana de São } \\
\text { Paulo }\end{array}$ & 5 & $17 \%$ & Vale do Paraíba Paulista & 2 & $7 \%$ \\
\hline Itapetininga & 3 & $10 \%$ & Araraquara & 1 & $3 \%$ \\
\hline Marília & 3 & $10 \%$ & $\begin{array}{l}\text { Macro Metropolitana } \\
\text { Paulista }\end{array}$ & 1 & $3 \%$ \\
\hline São José do Rio Preto & 3 & $10 \%$ & Presidente Prudente & 1 & $3 \%$ \\
\hline
\end{tabular}

Fonte: Elaborado pelo Autor

A Tabela 6 lista os 29 municípios benchmarking geral classificados por cluster. Em dados de 2010 eles somavam população de 3.401.861,00 habitantes, com população mediana de 40 mil habitantes.

Tabela 6 - Municípios Benchmarking Geral

\begin{tabular}{|c|c|c|c|c|}
\hline Município & Pop & Cluster & Mesorregião & Micorregião \\
\hline Espírito Santo do Pinhal & 41907 & I & Campinas & São João da Boa Vista \\
\hline Piedade & 52143 & I & Macro Metropolitana Paulista & Piedade \\
\hline Potim & 19397 & I & Vale do Paraíba Paulista & Guaratinguetá \\
\hline Ribeirão Branco & 18269 & I & Itapetininga & Capão Bonito \\
\hline Santa Cruz das Palmeiras & 29932 & I & Campinas & Pirassununga \\
\hline São Joaquim da Barra & 46512 & I & Ribeirão Preto & São Joaquim da Barra \\
\hline Socorro & 36686 & I & Campinas & Amparo \\
\hline Capão Bonito & 46178 & II & Itapetininga & Capão Bonito \\
\hline Franca & 318640 & II & Ribeirão Preto & Franca \\
\hline Francisco Morato & 154472 & ॥ & Metropolitana de São Paulo & Franco da Rocha \\
\hline Franco da Rocha & 131604 & II & Metropolitana de São Paulo & Franco da Rocha \\
\hline Santa Bárbara d'Oeste & 180009 & ॥ & Campinas & Campinas \\
\hline Santo André & 676407 & ॥ & Metropolitana de São Paulo & São Paulo \\
\hline São Vicente & 332445 & II & Metropolitana de São Paulo & Santos \\
\hline Tupã & 63476 & $\|$ & Marília & Tupã \\
\hline Álvaro de Carvalho & 4650 & III & Marília & Marília \\
\hline Estiva Gerbi & 10044 & III & Campinas & Moji-Mirim \\
\hline Itobi & 7546 & III & Campinas & São João da Boa Vista \\
\hline Nova Canaã Paulista & 2114 & III & São José do Rio Preto & Jales \\
\hline Riversul & 6163 & III & Itapetininga & Itapeva \\
\hline Santa Salete & 1447 & III & São José do Rio Preto & Jales \\
\hline Santo Antônio do Jardim & 5943 & III & Campinas & São João da Boa Vista \\
\hline Turmalina & 1978 & III & São José do Rio Preto & Fernandópolis \\
\hline Bastos & 20445 & IV & Marília & Tupã \\
\hline Itápolis & 40051 & IV & Araraquara & Araraquara \\
\hline Rosana & 19691 & IV & Presidente Prudente & Presidente Prudente \\
\hline São José dos Campos & 629921 & IV & Vale do Paraíba Paulista & São José dos Campos \\
\hline Sumaré & 241311 & IV & Campinas & Campinas \\
\hline Suzano & 262480 & IV & Metropolitana de São Paulo & Moji das Cruzes \\
\hline
\end{tabular}

Fonte: Elaborado pelo autor 
Entre os municípios classificados como benchmarking, há alguns que são fronteiriços, ou que compartilham as mesmas microrregiões. São os casos de:

- Santa Bárbara d'Oeste e Sumaré na microrregião de Campinas;

- Ribeirão Branco e Capão Bonito na microrregião de Capão Bonito;

- Francisco Morato e Franco da Rocha na microrregião de Franco da Rocha;

- Nova Canaã Paulista e Santa Salete na microrregião de Jales;

- Espírito Santo do Pinhal, Itobi e Santo Antônio do Jardim na microrregião de São João da Boa Vista;

- Bastos e Tupã na microrregião de Tupã;

Tal fato pode sinalizar a presença de fatores ambientais que influência a prática pública na promoção de desenvolvimento local. Também corrobora a questão de escala apontada por Vainer (2001) e Martins, Vaz e Caldas (2010). A escala do desenvolvimento local pode então ultrapassar o limite político do município. Podendo ser um conjunto de cidades que historicamente se desenvolveram em conjunto.

\section{CONSIDERAÇÕES FINAIS}

Este estudo teve como objetivo avaliar a eficiência dos munícipios do estado de São Paulo em promover desenvolvimento local. Para isso, foi construído um indicador utilizando Análise Envoltória de Dados com base em um modelo com retorno variável de escala e orientação para output. Além disso, para mitigar as diferenças entre municípios no Estado de São Paulo, ainda foi feita uma análise de Cluster, agrupando em quatro perfis de municípios com base nas principais fontes de receita:

- O Cluster I possui o maior número de municípios (40\% dos Municípios avaliados), as suas principais fontes de receita vem do repasse da cota de ICMS e do Fundo de Participação Municipal.

- O Cluster II foi composto por 150 municípios, representado 23\% do total analisado. São municípios normalmente de regiões metropolitanas, com importante perfil de arrecadação de tributos municipais, em específico IPTU e ISSQN.

- O Cluster III possui municípios pequenos e com dependência do repasse federal do Fundo de Partição Municipal, sendo $20 \%$ do total analisado.

- O Cluster IV reúne 74 municípios com forte participação nas receitas da cota parte de ICMS. São Municípios com forte perfil econômico e tem representantes em todas as mesorregiões do estado.

Ao todo, foram encontrados 29 municípios eficientes simultaneamente em todas as dimensões de desenvolvimento local avaliadas. Neste aspecto, há destaque para a Mesorregião de Campinas. Entre os 29 municípios benchmarking geral 28\% estão nesta mesorregião. Outro resultado que emana do estudo é a presença de municípios limítrofes, corroborando que a escala do Local ultrapassa o limite político municipal.

Sugere-se como estudo futuro investigar de maneira mais aprofundada os 29 municípios classificados como eficientes nas quatro dimensões. Compreender o que os diferencia dos demais pode ser útil para criação de políticas públicas que valorizem a eficiência no uso do dinheiro público. Além disso, sugere-se a utilização de técnicas paramétricas que permitam a extrapolação dos dados. 


\section{AGRADECIMENTO}

Os autores agradecem o apoio financeiro concedido pela Fundação de Amparo à Pesquisa do Estado de São Paulo (FAPESP) e Coordenação de Aperfeiçoamento de Pessoal de Nível Superior (CAPES), mediante processo n. 2014/20751-3.

\section{REFERÊNCIAS}

ADLER, N.; YAZHEMSKY, E.; TARVERDYAN, R. A framework to measure the relative socio-economic performance of developing countries. Socio-Economic Planning Sciences, v. 44, n. 2, p. 73-88, jun. 2010.

AFONSO, A.; SCHUKNECHT, L.; TANZI, V. Income distribution determinants and public spending efficiency. The Journal of Economic Inequality, v. 8, n. 3, p. 367-89, jun. 2010.

AFONSO, A.; FERNANDES, S. Assessing and explaining the relative efficiency of local government: evidence for portuguese municipalities. Social Science Electronic Publishing, nov. 2005. Disponível em: https:// papers.ssrn.com/sol3/papers.cfm?abstract_id=849247.

BOGONI, N.; HEIN, N.; BEUREN, I. Análise da relação entre crescimento econômico e gastos públicos nas maiores cidades da região Sul do Brasil. Revista de Administração Pública, Rio de Janeiro, v. 45, n. 1, p. 159-79, jan./fev. 2012.

BORGES, C. M. Desenvolvimento local e avaliação de políticas públicas: análise de viabilidade para construção de um índice de desenvolvimento local para o município de São José do Rio Preto. 2007. Dissertação (Mestrado em Administração de Organizações) - Faculdade de Economia, Administração e Contabilidade de Ribeirão Preto, Universidade de São Paulo, Ribeirão Preto, SP, 2007.

CARVALHO, J. B. et al. Desempenho da produção agropecuária dos municípios pertencentes ao Escritório de Desenvolvimento Rural de Andradina, SP, Brasil. Interações, Campo Grande, MS, v. 18, n. 2, p. 171-84, abr./jun. 2017.

CHARNES, A.; COOPER, W. W.; RHODES, E. Measuring the efficiency of decision making units. European Journal of Operational Research, v. 2, n. 6, p. 429-44, nov. 1978.

CHOJNICKI, Z. Socio-economic development and its axiological aspects. Quaestiones Geographicae, v. 29, n. 2, p. 7-17, 2010.

COLLUCI, L. O impacto na ponderação do peso da Prova Brasil e do indicador de rendimento no perfil das escolas municipais do ensino fundamental consideradas eficientes pela técnica DEA em transformar investimento financeiro em desempenho no IDEB em 2011. 2014. Dissertação (Mestrado em Administração de Organizações) - Universidade de São Paulo, Ribeirão Preto, SP, 2014.

CRUZ, N. F.; MARQUES, R. C. Revisiting the determinants of local government performance. Omega, v. 44, p. 91-103, abr. 2014.

DYSON, R. G. et al. Pitfalls and protocols in DEA. European Journal of Operational Research, v. 132, n. 2, p. 245-59, jul. 2001.

FARIA, F.; JANNUZZI, P.; SILVA, S. Eficiência dos gastos municipais em saúde e educação: uma investigação através da análise envoltória no estado do Rio de Janeiro. Revista de Administração Pública, Rio de Janeiro, v. 42, n. 1, p. 155-77, jan./fev. 2008. 
FILIPPIM, E.; ROSSETTO, A.; ROSSETTO, C. Abordagens da administração pública e sua relação com o desenvolvimento em um contexto regional: o caso do Meio-Oeste Catarinense. Cadernos EBAPE.BR, Rio de Janeiro, v. 8, n. 4, p. 734-52, dez. 2010.

FRAINER, D. M. et al. Uma aplicação do Índice de Desenvolvimento Sustentável aos municípios do estado de Mato Grosso do Sul. Interações, Campo Grande, MS, v. 18, n. 2, p. 145-56, abr./jun. 2017.

GAROFOLI, G. Local development in Europe: theoretical models and international comparisons. European Urban and Regional Studies, v. 9, n. 3, p. 225-39, jul. 2002.

GOLANY, B.; ROLL, Y. An application procedure for DEA. Omega, v. 17, n. 3, p. 237-50, 1989.

LINDBLAD, M. R. Performance measurement in local economic development. Urban Affairs Review, v. 41, n. 5, p. 646-72, maio 2006.

LU, W.-M.; LO, S.-F. Constructing stratifications for regions in China with sustainable development concerns. Quality \& Quantity, v. 46, n. 6, p. 1807-23, ago. 2012.

MARTINELLI, D. P.; JOYAL, A. Desenvolvimento local e o papel das pequenas e médias empresas. São Paulo: Manole, 2004.

MARTINS, S. Desenvolvimento local: questões conceituais e metodológicas. Interações, Campo Grande, MS, v. 3, n. 5, p. 51-9, set. 2002.

MARTINS, R.; VAZ, J. C.; CALDAS, E. L. A gestão do desenvolvimento local no Brasil: (des)articulação de atores, instrumentos e território. Revista de Administração Pública, Rio de Janeiro, v. 44, n. 3, p. 559-90, maio/jun. 2010.

MURIAS, P.; MARTINEZ, F.; MIGUEL, C. An economic wellbeing index for the spanish provinces: a data envelopment analysis approach. Social Indicators Research, v. 77, n. 3, p. 395-417, jul. 2006.

NEL, E. Local economic development : a review and assessment of its current status in South Africa. Urban Studies, v. 38, n. 7, p. 1003-24, 2001.

OLIVEIRA, B. G. Eficiência da gestão pública em promover desenvolvimento local de regiões produtoras de sucroenergia: uma proposta utilizando Análise Envoltória de Dados. 2015. Dissertação (Mestrado em Administração de Organizações) - Universidade de São Paulo, Ribeirão Preto, SP, 2015.

RAMOS, M.; SCHABBACH, L. O estado da arte da avaliação de políticas públicas: conceituação e exemplos de avaliação no Brasil. Revista de Administração Pública, Rio de Janeiro, v. 46, n. 5, p. 1271-94, set./out. 2012.

REIG-MARTÍNEZ, E. Social and economic wellbeing in Europe and the mediterranean basin: building an enlarged human development indicator. Social Indicators Research, v. 111, n. 2, p. 527-47, mar. 2013.

SANTOS, N. B. et al. Desenvolvimento e crescimento econômico das macrorregiões de Mato Grosso nos anos 2005 e 2013. Interações, Campo Grande, MS, v. 18, n. 3, p. 169-82, jul./set. 2017.

SCARPIN, J. E.; SLOMSKI, V. Estudo dos fatores condicionantes do índice de desenvolvimento humano nos municípios do estado do Paraná: instrumento de controladoria para a tomada de decisões na gestão governamental. Revista de Administração Pública, Rio de Janeiro, v. 41, n. 5, p. 909-33, set./out. 2007.

NOVAIS, L. F. A economia paulista pós-crise de 2008: papel da indústria de transformação no cenário recente. Ensaio \& Conjuntura, São Paulo, ago. 2017. Disponível em: http://www.seade.gov.br/produtos/ midia/2017/09/Desafios_-economia_paulista.pdf 
SECRETÁRIA DE PLANEJAMENTO E DESENVOLVIMENTO REGIONAL (SEADE). Informações dos Municípios Paulistas -IMP. 2013. Disponível em: http://www.imp.seade.gov.br/frontend/\#/ Acesso em: 12 set. 2019.

SOUZA, C. Governos locais e gestão de políticas sociais universais. São Paulo em Perspectiva, São Paulo, v. 18, n. 2, p. 27-41, abr./jun. 2004.

SOUZA, V. et al. Uso da análise envoltória de dados para mensuração da sustentabilidade de hotéis-fazenda em Pernambuco. Interações, Campo Grande, MS, v. 18, n. 1, p. 41-57, jan./mar. 2017.

TESOURO NACIONAL. Finanças do Brasil - estados e municípios. 2013. Disponível em: https://www. tesourotransparente.gov.br/temas/estados-e-municipios/boletim-de-financas-dos-entes-subnacionais Acesso em: 12 set. 2019.

TRISTÃO, J. A. M. A administração tributária dos municípios brasileiros- uma avaliação do desempenho da arrecadação. 2003. Tese (Doutorado em Administração) - Fundação Getúlio Vargas, São Paulo, 2003.

VAINER, C. B. As escalas do poder e o poder das escalas: o que pode o poder local? In: ENCONTRO NACIONAL DA ANPUR, 9., 2001, Rio de Janeiro. Anais [...] Rio de Janeiro: ANPUR, 2001. p. 140-51.

WILSON, P. Embracing locality in local economic development. Urban Studies, v. 32, n. 4-5, p. 645-58, maio 1995.

WONG, C. Developing indicators to inform local economic development in England. Urban Studies, v. 39, n. 10, p. 1833-63, 2002.

\section{Sobre os autores:}

Bruno Garcia Oliveira : Doutorado em andamento na Universidade de São Paulo (FEA-RP/ USP), Mestre pela Universidade de São Paulo (FEA-RP USP) na área de Administração de Organizações. Graduado em Administração pela Universidade Federal de Goiás (UFG-CAC). Desenvolve pesquisa sobre capacidade dinâmica, desenvolvimento local e setor sucroenergético. Professor do Curso de Administração da Universidade Federal de Goiás - Regional Catalão (CGEN-UFG/RC). E-mail: brunogarcia@usp.br, Orcid: http://orcid.org/0000-0003-4257-5542

Lara Bartocci Liboni: Livre-docente pela FEARP/USP, Faculdade de Economia, Administração e Contabilidade de Ribeirão Preto, Universidade de São Paulo. Pós-doutora pela UNESP (FEB-Bauru). Doutora-Phd e mestre em Administração pela Faculdade de Economia, Administração e Contabilidade da Universidade de São Paulo (FEA/USP). Graduada em Administração pela FEARP/USP. Professora Associada do Departamento de Administração, da FEARP/USP. E-mail: laraliboni@fearp.usp.br, Orcid: http://orcid.org/0000-0002-4729-7943 